\title{
Erratum to: Early-onset bipolar disorder: how about visual-spatial skills and executive functions?
}

\author{
Sara Lera-Miguel · Susana Andrés-Perpiñá • \\ Rosa Calvo • Mar Fatjó-Vilas · Lourdes Fañanás · \\ Luisa Lázaro
}

Published online: 19 January 2011

(c) Springer-Verlag 2011

\section{Erratum to: Eur Arch Psychiatry Clin Neurosci DOI 10.1007/s00406-010-0169-z}

Unfortunately, the name of one of the authors was published with errors. The correct list is given here.

S. Lera-Miguel, S. Andrés-Perpiñá, R. Calvo, L. Lázaro Department of Child and Adolescent Psychiatry and Psychology, Hospital Clínic de Barcelona, Barcelona, Spain e-mail: slera@clinic.ub.es

S. Andrés-Perpiñá, R. Calvo, M. Fatjó-Vilas, L. Fañanás, L. Lázaro

Centro de Investigación Biomédica en Red de Salud Mental CIBERSAM, Barcelona, Spain

The online version of the original article can be found under doi:10.1007/s00406-010-0169-z.

S. Lera-Miguel $(\varangle) \cdot$ S. Andrés-Perpiñá · R. Calvo · L. Lázaro Department of Child and Adolescent Psychiatry and Psychology, Hospital Clínic de Barcelona, Barcelona, Spain

e-mail: slera@clinic.ub.es

S. Andrés-Perpiñá $\cdot$ R. Calvo · M. Fatjó-Vilas · L. Fañanás ·

L. Lázaro

Centro de Investigación Biomédica en Red de Salud Mental CIBERSAM, Barcelona, Spain

S. Andrés-Perpiñá · L. Lázaro

Institut d'Investigacions Biomèdiques August Pi i Sunyer

IDIBAPS, Barcelona, Spain

M. Fatjó-Vilas · L. Fañanás

Department of Biologia Animal, Facultat de Biologia,

Universitat de Barcelona, Barcelona, Spain

M. Fatjó-Vilas · L. Fañanás

Institut de Biomedicina de la Universitat de Barcelona

IBUB, Barcelona, Spain
S. Andrés-Perpiñá, L. Lázaro

Institut d'Investigacions Biomèdiques August Pi i Sunyer IDIBAPS, Barcelona, Spain

M. Fatjó-Vilas, L. Fañanás

Department of Biologia Animal, Facultat de Biologia, Universitat de Barcelona, Barcelona, Spain

M. Fatjó-Vilas, L. Fañanás

Institut de Biomedicina de la Universitat de Barcelona IBUB, Barcelona, Spain 